\title{
Natural and totally synthetic receptors in the innovative design of HPLC chiral stationary phases*
}

\author{
Francesco Gasparrini ${ }^{\ddagger}$, Ilaria D’Acquarica, Domenico Misiti, \\ Marco Pierini, and Claudio Villani
}

\begin{abstract}
Dipartimento di Studi di Chimica e Tecnologia delle Sostanze Biologicamente Attive, Università "La Sapienza", P. le A. Moro 5, 00185 Roma, Italy
\end{abstract}

\begin{abstract}
An overview is presented of the experimental results obtained by our group in the design, realization, and applications of high-performance liquid chromatography (HPLC) enantioselective stationary phases based on surface-linked macrocyclic receptors. Macrocyclic synthetic receptors and macrocyclic glycopeptide receptors have been grafted on silica particles, and the resulting chiral stationary phases are used to monitor differential associations of the enantiomers of selected chiral guests with the immobilized hosts. Practical implications of the chiral phases in enantioselective analysis and in the study of recognition events are highlighted.
\end{abstract}

\section{INTRODUCTION}

Progress in various fields related to organic chemistry has always been linked to parallel advancements in separation technology. When chiral compounds of natural or synthetic origin are considered, the advent of high-performance liquid chromatography (HPLC) and gas chromatography (GC) chiral stationary phases has greatly facilitated the development of new procedures for the preparation of enantiomerically pure compounds. From the analytical point of view, the determination of enantiomeric composition is of great importance in the study of chiral compounds. Synthetic procedures, and characterization and practical use of chiral synthons, auxiliaries, agrochemicals, and pheromones require stereochemical methodologies that are fast, simple, and accurate. The need of advanced enantioselective separation technologies is even more evident in those fields connected with chiral compounds interacting with living systems. It is well recognized that the two enantiomers of a chiral drug often show significant differences in their activity, pharmacokinetics, pharmacodynamics, and potential adverse drug reaction. For this reason, stringent stereochemical characterizations of drug substances are now required by regulatory agencies [1]. In spite of the increasing scientific activity around enantioselective preparation and analysis of chiral compounds, the chromatographic resolution of enantiomeric molecules still remains a great challenge in the field of separation science. Enantioselective chromatographic systems rely on suitable chiral selectors in enantioenriched form, usually anchored to a support matrix and forming the chiral stationary phase (CSP). The system then manifests its enantioselectivity through the differential interaction of the chiral selector with the two enantiomeric solutes to be resolved. This enantioselectivity is thermodynamic in origin and is due to the different stability of the diastereomeric species formed by the two enantiomers with the immobilized selector. In the absence of achiral inter-

\footnotetext{
* Pure Appl. Chem. 75, 141-419 (2003). An issue of reviews and research papers based on lectures presented at the $23^{\text {rd }}$ IUPAC International Symposium on the Chemistry of Natural Products, Florence, Italy, 28 July-2 August 2002 on the theme of natural products.

¥Corresponding author: E-mail: francesco.gasparrini@uniroma1.it
} 
actions, chromatographically derived enantioselectivity $\alpha$ is related to the Gibbs energy difference by eqs. 1 and 2:

$$
\begin{aligned}
& \delta \Delta G_{R, S}=\Delta G_{R}-\Delta G_{S}=-R T \ln \alpha_{R, S} \\
& \delta \Delta H_{R, S}-T \delta \Delta S_{R, S}=-R T \ln \alpha_{R, S}
\end{aligned}
$$

where $\delta \Delta G_{R, S}, \delta \Delta H_{R, S}$, and $\delta \Delta S_{R, S}$ are the difference in the Gibbs free energy, enthalpy and entropy between the two enantiomers and the system selector, $R$ is the gas constant and $T$ the absolute temperature. Typical $\delta \Delta G_{R, S}$ values encountered in modern HPLC enantioresolution are between 0.2 and $1.4 \mathrm{kcal} / \mathrm{mol}$ at room temperature [2].

In spite of the progress obtained in the understanding of recognition events at molecular level, the de novo design of chiral selectors that yield large selectivities for a given set of enantiomeric solutes is not an easy task. Rather, the incorporation of some structural features into a molecular entity usually results in highly enantioselective selectors and chiral stationary phases: (a) presence of functional groups with high directionality, e.g., amide fragments with H-bond donor/acceptor capability; (b) limited conformational flexibility; and (c) absence of nonproductive interaction sites.

\section{RESULTS}

Totally synthetic chiral stationary phases that incorporate highly preorganized, receptor-like chiral selectors have been prepared and used in the study of enantiorecognition processes characterized by high selectivities $\left(\alpha=12, \delta \Delta G_{R, S}=1.5 \mathrm{kcal} / \mathrm{mol}\right.$ at $\left.25^{\circ} \mathrm{C}\right)$. These CSPs were prepared by the radical addition of thiol groups on the surface of 3-mercaptopropyl silica across the pendant allyl groups of the chiral receptors. Typical grafting rates were $0.15 \mu \mathrm{mol}$ of receptor per gram of silica.

$\mathrm{A} \mathrm{C}_{3}$ symmetric, basket-like receptor incorporating three $O$-allylated L-tyrosine chiral units at the periphery and a flat 1,3,5-trimercaptobenzene portion closing the bottom of the structure (Fig. 1) was linked to 3-mercaptopropyl silica gel to afford the corresponding CSP1 [3]. This receptor-like stationary phase showed extreme enantioselectivity in organic solvents for fully protected amino acids with either the $\mathrm{N}$ - or C-terminal units carrying a bulky group. On CSP1 we recorded $\delta \Delta G_{R, S}$ values up to $2.5 \mathrm{kcal} / \mathrm{mol}$ in favor of the L enantiomers of protected amino acids, with a marked dependence of selectivity on the amino acid side chain shape and functionality and on the nature of protective groups at carboxy- and amino-terminus. Large affinities and selectivities were observed for branched or hydroxylated side chains and for short $\mathrm{C}$-terminus alkylamides and $\mathrm{N}$-cyclopropanoyl residues. ${ }^{1} \mathrm{H}$ NMR data collected on systems comprising the unbound receptor and N-Boc amino acid methylamides or N-Moc amino acid tbutyl esters were consistent with a recognition mechanism positioning the small $\mathrm{CO}-\mathrm{NH}-$ methyl or $\mathrm{NH}-\mathrm{COOMe}$ termini inside the macrocyclic cavity, with the partners held together in the complexes by a network of H-bonds [4].
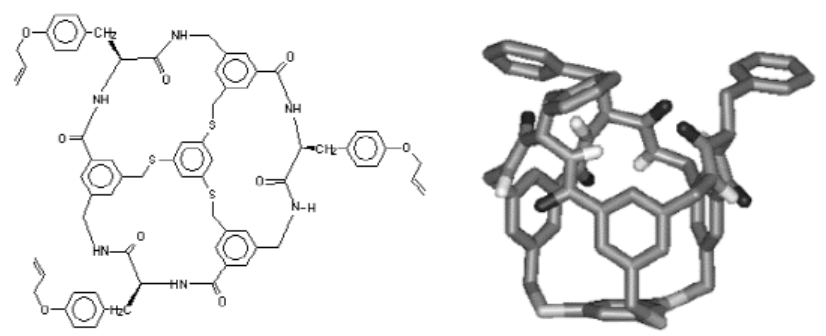

Fig. 1 Left: Synthetic $\mathrm{C}_{3}$ chiral receptor leading to CSP1 after immobilization on $\gamma$-mercaptopropyl silica. Right: Polytube model of a low-energy structure (tyrosil side chains are omitted). 

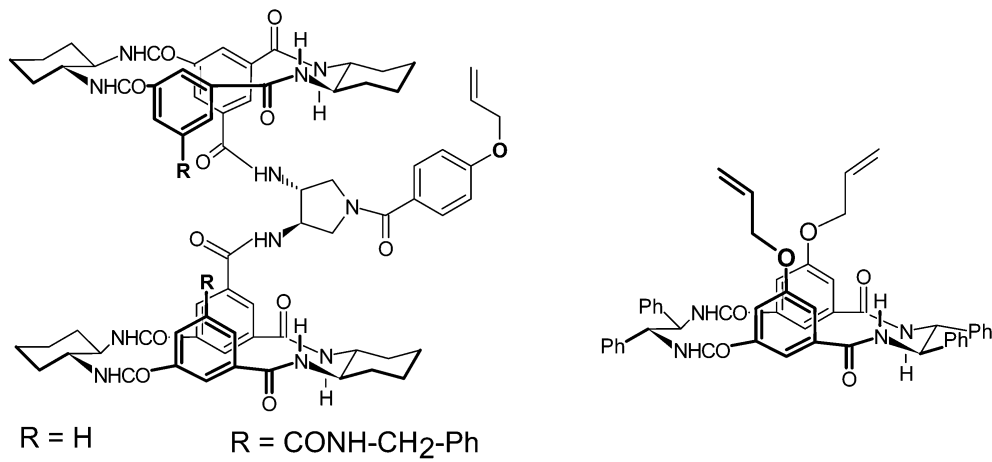

Fig. 2 Left: Synthetic $\mathrm{C}_{2}$ chiral receptors leading to $\mathrm{CSP} 2(\mathrm{R}=\mathrm{H})$ and $\mathrm{CSP} 3\left(\mathrm{R}=\mathrm{CONH}-\mathrm{CH}_{2}-\mathrm{Ph}\right)$ after immobilization on $\gamma$-mercaptopropyl silica. Right: Synthetic $\mathrm{C}_{2}$ chiral minireceptor leading to $\mathrm{CSP} 4$ after immobilization on $\gamma$-mercaptopropyl silica.

A second kind of receptor-like selectors were provided by two $\mathrm{C}_{2}$ symmetric bis-armed molecules derived from identical tetra-amide subunits [constructed from $(R, R)$-1,2-diaminocyclohexane and phthalic or trimesic acid], connected to a $N$-(4-allyloxy benzoylated)-( $R, R)-2,3$-diaminopyrrolidine [5]. After surface immobilization on silica gel (CSP2 and CSP3, Fig. 2, left), these $\mathrm{C}_{2}$ receptors showed large selectivity for some fully protected tripeptides. Their selectivity was dependent on both the sequence and stereochemistry of the peptidic fragments. The components of a small peptide library (the eight stereoisomers of the tripeptide Acetyl-Pro-Val-Gln propylamide) were individually prepared and their affinities for the immobilized receptors were evaluated using $\mathrm{CH}_{2} \mathrm{Cl}_{2}$-based eluents. Both phases behaved similarly, in that they showed high affinity for a single stereoisomer only (CSP2: D,L,D sequence CSP3: L,L,D sequence). In addition, these large affinities were accompanied by large values of enantioselectivities $\left(\delta \Delta G_{D, L, D / L, D, L}=-1.6 \mathrm{kcal} / \mathrm{mol}\right.$ and $\delta \Delta G_{L, L, D / D, D, L}=-1.7 \mathrm{kcal} / \mathrm{mol}$ for CSP2 and CSP3, respectively).

Interestingly, analysis of the association process of the tripeptides with the immobilized receptors in the $25-85{ }^{\circ} \mathrm{C}$ temperature range showed that the loosely bound stereoisomers had largely positive enthalphy and entropy of adsorption, while the tightly bound stereoisomers had negative enthalpy and close to zero entropy of adsorption. Theses findings where interpreted in terms of an entropydriven binding process dominated by solvation-desolvation effects, a situation that is likely to occur when the interacting partners come in contact by sharing large surfaces and establishing multipoint interactions.

A simplified version of the bis-armed macrocyclic chiral receptor was designed by using a single macrocyclic unit having the more sterically demanding $(R, R)$-1,2-diphenylethylendiamine in place of the cyclic diamine (Fig. 2, right). The new receptor, after surface immobilization on HPLC silica particles to give CSP4, showed extreme enantioselectivities towards simple amino acid hexyl amides having the $\pi$-basic 3,5-dinitrobenzoyl group at the $\mathrm{N}$-terminal ends. With amino acid having side chains of 3 to 4 carbon atoms, either linear or $\beta$-branched, $\delta \Delta G_{R, S}$ value approaching $3.0 \mathrm{kcal} / \mathrm{mol}$ were recorded in organic solvents [6].

The naturally occurring glycopeptide antibiotics, like teicoplanin and vancomycin, offer more complex molecular architectures compared to the totally synthetic receptors used in CSPs 1-4. Glycopeptide antibiotics and related molecules have a large, cyclic peptide backbone rich in aromatic fragments, surrounded by polar and ionizable groups and carrying carbohydrate fragments at the macrocycle periphery. The cyclic peptide backbone has a conformationally rigid, cup-shaped architecture, with the aromatic fragments rigidly interlocked in a well-defined stereochemical disposition. These naturally occurring macrocycles have antibiotic activity against gram-positive bacteria because they bind to the terminal D-Ala-D-Ala dipeptide of peptidoglycans precursors and prevent the construction of bac- 


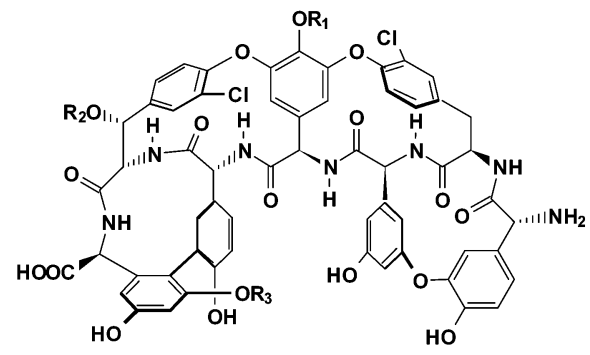

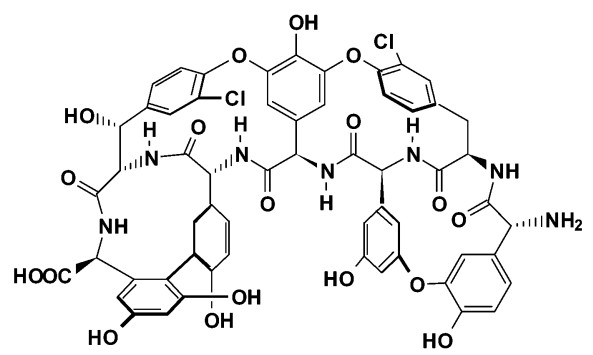

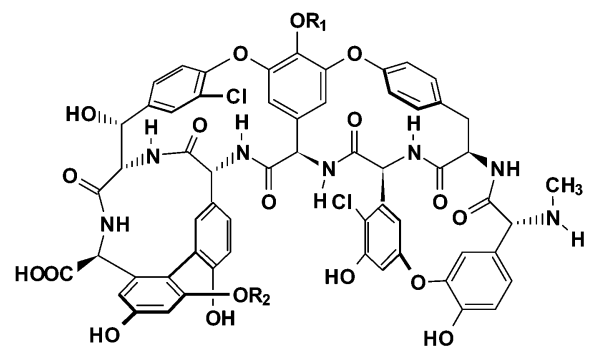

teicoplanin

$\mathbf{R}_{\mathbf{1}}, \mathbf{R}_{\mathbf{2}}, \mathbf{R}_{\mathbf{3}}=$ sugar

teicoplanin aglycone
A-40,926

$\mathbf{R}_{\mathbf{1}}, \mathbf{R}_{\mathbf{2}}$ = sugar

$\mathrm{R}_{1}$ has a carboxyl function

Fig. 3 Structures of macrocyclic receptors of the teicoplanin family (CSP5, teicoplanin; CSP6, teicoplanin aglycone; CSP7, A-40,926).

terial cell walls. Teicoplanin, vancomycin, and related molecules have been recently introduced into the vast repertoire of selectors for HPLC CSPs, as their molecular structure is well suited for enantioselective recognition as well as for immobilization on a solid support $[7,8]$. Three closely related stationary phases of this kind have been recently prepared by our group (Fig. 3).

They contain immobilized teicoplanin, its aglycone and the A40-926 macrocyclic peptide as chiral selectors [9-16]. A new process that affords stable chiral phases was developed for the surface immobilization of these compounds. Aminopropyl silica was activated with 1,6-diisocyanatohexane to yield a reactive intermediate material presenting on the silica surface residual, pendant isocyanate groups. These were used in the last step to immobilize the chiral receptor through their free amino groups, giving the final CSPs with typical receptor loadings of $1.5 \mu \mathrm{mol} / \mathrm{g}$ of silica.

The immobilized teicoplanin turned out to be extremely efficient in the enantiomeric resolution of permanently charged species like carnitines [9] and ruthenium(II) octahedral complexes with bidentate aromatic ligands [15-16] and of ionizable chiral compounds having a carboxylic fragment. A distinctive feature of these new chiral stationary phases is their ability to separate the enantiomers of $\alpha$-, $\beta$ - and $\gamma$-amino acids using simple water-alcohol mobile phases. While the enantiorecognition ability observed for chiral species carrying free carboxylic moieties was not surprising considering the es- 
tablished propensity of the glycopeptides to bind the natural target at the carboxy terminus, the large selectivity for the enantiomers of octahedral ruthenium complexes was totally unexpected. Indeed, ruthenium(II) complexes with ancillary ligands like 2,2'-bipyridine, 1,10-phenanthroline, and 4,7-diphenyl1,10-phenanthroline showed enantioselective associations with immobilized teicoplanin and teicoplanin aglycone, with the extent of discrimination between the $\Delta$ and $\Lambda$ enantiomers increasing with the size of the flat portion of the aromatic ligands and decreasing with steric bulk at the distal positions. The potentiality of immobilized glycopeptide antibiotics in the preparative resolution of otherwise difficult-to-resolve organic and organometallic molecules was also demonstrated $[14,16]$.

\section{CONCLUSION}

Chiral stationary phases with receptor-like enantioselectivity represent powerful tools in the study of the intermolecular interactions that drive and regulate host-guest associations at molecular levels. New stationary phases incorporating chiral-pool molecular receptors (e.g., the glycopeptides family) showed large enantioselectivities in water-rich media for charged and polar species. Their mode of action is largely unexplored, and it can be expected that they will give structural and energetic information about chiral systems where association is driven by hydrophobic forces. On the side of totally synthetic receptors, the preparation of simplified structures that can be easily obtained in large amounts and with tailored enantioselectivities for specific compound classes has immediate implications in the study of recognition mechanisms in organic solvents as well in the facile preparative isolations of pure enantiomers.

\section{ACKNOWLEDGMENT}

Part of this work was supported by funds from MIUR (Contract No. 2001033797_004) and from Centro di Eccellenza BEMM.

\section{REFERENCES}

1. S. Ahuja (Ed.). Chiral Separations: Applications and Technology, American Chemical Society, Washington, DC (1997).

2. F. Gasparrini, D. Misiti, C. Villani. J. Chromatogr. A 724, 79 (1996).

3. F. Gasparrini, D. Misiti, C. Villani, A. Borchardt, M. T. Burger, W. C. Still. J. Org. Chem. 60, 4314 (1995).

4. F. Gasparrini, D. Misiti, C. Villani. J. Chromatogr. A 906, 35 (2001).

5. F. Gasparrini, D. Misiti, C. Villani. H. Wennemers, W. C. Still. J. Org. Chem. 62, 8221 (1997).

6. F. Gasparrini, D. Misiti, M. Pierini, C. Villani. Org. Lett. 4, 3993 (2002).

7. D. W. Armstrong, Y. Tang, S. Chen, Y. Zhou, C. Bagwill, J.-R. Chen. Anal. Chem. 66, 1473 (1994).

8. T. J. Ward and A. B. Farris. J. Chromatogr. A 906, 73 (2001).

9. I. D'Acquarica, F. Gasparrini, D. Misiti, C. Villani, A. Carotti, S. Cellamare, S. Muck. J. Chromatogr. A 875, 145 (1999).

10. A. Berthod, X. Chen, J. P. Kullman, D. W. Armstrong, F. Gasparrini, I. D'Acquarica, C. Villani, A. Carotti. Anal. Chem. 72, 1767 (2000).

11. A. Berthod, T. Yu, J. P. Kullman, D. W. Armstrong, F. Gasparrini, I. D’Acquarica, D. Misti, A. Carotti. J. Chromatogr. A 897, 113 (2000).

12. G. Cancelliere, I. D’Acquarica, F. Gasparrini, D. Misiti, C. Villani. Pharma. Sci. Technol. Today 2, 484 (1999).

13. I. D'Acquarica, F. Gasparrini, D. Misiti, G. Zappia, C. Cimarelli, G. Calmieri, A. Carotti, S. Cellamare, C. Villani. Tetrahedron: Asymmetry 11, 2375 (2000). 
14. S. Alcaro, I. D'Acquarica, F. Gasparrini, D. Misiti, M. Pierini, C. Villani. Tetrahedron: Asymmetry 13, 69 (2002).

15. F. Gasparrini, I. D’Acquarica, J. G. Vos, C. M. O’Connor, C. Villani. Tetrahedron: Asymmetry 11, 3535 (2000).

16. W. R. Browne, C. M. O'Connor, C. Villani, J. G. Vos. Inorg. Chem. 40, 5461 (2001). 